\title{
Should Epidermal Growth Factor Receptor-Tyrosine Kinase Inhibitor Be Continued beyond Progressive Disease?
}

\author{
Young Hak Kim Akiko Fukuhara Michiaki Mishima \\ Department of Respiratory Medicine, Graduate School of Medicine, Kyoto \\ University, Kyoto, Japan
}

\section{Key Words}

Epidermal growth factor receptor-tyrosine kinase inhibitor . Progressive disease .

Non-small cell lung cancer - EGFR mutation · Acquired resistance · Continuation

\begin{abstract}
Epidermal growth factor receptor-tyrosine kinase inhibitor (EGFR-TKI) is almost exclusively effective in patients with activating EGFR mutations, and median time to progression in such patients is generally up to 12 months. Usually, treatment with EGFRTKI is terminated when disease progression is confirmed; however, acute exacerbation after the withdrawal of EGFR-TKI has been reported. In this paper, we report a case of a 35-year-old patient whose disease rapidly progressed after discontinuation of gefitinib and then rapidly regressed after reintroduction of gefitinib. In addition, we summarize the cases of 3 other patients who could be safely treated with continued erlotinib in combination with pemetrexed after disease progression. Currently, the mechanism of acquired resistance is intensively investigated and a number of new agents, such as irreversible EGFR inhibitors or MET inhibitors, are under development; however, they are still unavailable in clinical setting. We believe that continuing EGFR-TKI treatment after disease progression should be an option in patients who previously responded to EGFRTKI under the present circumstances.
\end{abstract}

\section{Introduction}

Epidermal growth factor receptor-tyrosine kinase inhibitor (EGFR-TKI), such as gefitinib or erlotinib, has been approved for the treatment of non-small cell lung cancer (NSCLC) in many countries, and it is widely accepted that specific mutations in the ATPbinding site of EGFR are strongly associated with the response to EGFR-TKI. Patients with such mutations greatly benefit from EGFR-TKI; however, median time to 
progression is reportedly 7.7-13.3 months because of acquired resistance. Treatment with EGFR-TKI is generally terminated once disease progression is confirmed, although a previous report suggests acute exacerbation after withdrawal of EGFR-TKI. We report the case of a patient with NSCLC whose disease rapidly progressed after discontinuation of gefitinib and then restarted concurrently with cytotoxic chemotherapy. We also present the cases of 3 other patients who continued erlotinib after disease progression in combination with pemetrexed.

\section{Case Report}

A 35-year-old male former smoker, presenting with severe cough for the past several months, was referred to our hospital and was found to have miliary pulmonary nodules in both lungs. After admission, bronchoscopic examination was performed and histologic analysis revealed adenocarcinoma. He was diagnosed with lung adenocarcinoma with multiple lung, bone, and brain metastases. He complained of dyspnea and his Eastern Cooperative Oncology Group performance status was 3 . His attending physician recommended gefitinib considering his poor performance status, and the treatment was initiated. His disease markedly improved without severe toxicity, and he was discharged from our hospital 1 month after admission (fig. 1a); however, 7 months later, he developed meningitis carcinomatosa and the multiple pulmonary metastases were exacerbated (fig. 1b). Gefitinib was terminated and whole brain irradiation was immediately started; however, miliary pulmonary metastases rapidly progressed during the following 3 weeks (fig. 1c), and gefitinib was restarted concurrently with gemcitabine and vinorelbine. Ten days later, the miliary pulmonary metastases rapidly improved (fig. 1d), and he continued to receive combination chemotherapy with gemcitabine, vinorelbine, and gefitinib; however, 4 months later, his disease progressed again and he died. The EGFR mutational status was unknown, while the patient was alive, but was later revealed to be the wild type; however, such a great benefit of gefitinib in wild-type patients is hard to imagine [1]. It seems possible to assume that the result was a false negative. The analyzed tissue was old and very small, which may have influenced the accuracy of the analysis.

\section{Discussion}

In 2007, Riely et al. [2] reported the results of their interesting study. Ten patients with NSCLC, who initially responded but later acquired resistance to EGFR-TKI, gefitinib or erlotinib, had 18-fluoro-2-deoxy-D-glucose-positron emission tomography/computed tomography (PET/CT) and computed tomography (CT) at baseline, 3 weeks after stopping EGFR-TKI, and 3 weeks after restarting EGFR-TKI. Three weeks after stopping EGFR-TKI, there was a median 18\% increase in SUVmax and a 9\% increase in tumor diameter, and 7 out of 10 patients showed worsening lung cancer symptoms; however, 3 weeks after restarting the same EGFR-TKI, there was a median $4 \%$ decrease in SUVmax and $1 \%$ decrease in tumor diameter, and all 7 patients whose symptoms had worsened after stopping EGFR-TKI stabilized or had improved symptoms [2]. These results suggest that some tumor cells remain sensitive to EGFR-TKI after disease progression has been confirmed. A similar phenomenon has also been reported with imatinib for gastrointestinal stromal tumor [3].

To date, 4 patients have been receiving EGFR-TKI after disease progression in our hospital (table 1). All 4 patients had adenocarcinoma histology, and EGFR-active mutation was confirmed in 2 patients. Initial response to EGFR-TKI was partial response $(\mathrm{PR})$ in 3 patients and stable disease (SD) of long duration in 1 patient. Case 3 had previously received gefitinib and achieved PR. Pemetrexed was chosen for add-on chemotherapy except for the present case, and there were $1 \mathrm{PR}, 1$ long SD, and 1 
progressive disease (PD). Both case 1 and case 3 are currently being treated with the same regimen.

Collectively, it seems that some types of patients will clearly benefit from continuing EGFR-TKI beyond PD; however, it is needless to say that a randomized trial comparing chemotherapy with or without continuation of EGFR-TKI at the time of acquired resistance is crucial for confirmation.

When combining EGFR-TKI and chemotherapy, the best regimen for the EGFR-TKI partner with regard to both safety and efficacy is still unknown. Although previous large phase III studies did not show increased toxicity with the combination of carboplatin + paclitaxel or cisplatin + gemcitabine with EGFR-TKIs [4-7], multiple reports do not support the concurrent administration of vinorelbine and gefitinib because of severe toxicity $[8,9]$. In a recent study, on the other hand, the combination of erlotinib and pemetrexed was reported to be feasible and well tolerated [10].

Currently, a number of new agents, such as irreversible EGFR inhibitors or MET inhibitors, are under development to overcome acquired resistance to EGFR-TKI [11]; however, they are still unavailable in clinical setting. Under the present circumstances, continuing EGFR-TKI after disease progression should be an option in patients who previously responded to EGFR-TKI.

\section{Disclosure Statement}

No author has a financial relationship with a commercial entity that has an interest in the topic of this paper.

Table 1. Summary of patients who received EGFR-TKI beyond PD in combination with a cytotoxic agent

\begin{tabular}{|c|c|c|c|c|c|c|c|}
\hline Case & Age/sex & Histology & $\begin{array}{l}\text { EGFR } \\
\text { mutation }\end{array}$ & EGFR-TKI/response & $\begin{array}{l}\text { Response } \\
\text { duration }\end{array}$ & Add-on/response & $\begin{array}{l}\text { Response } \\
\text { duration } \\
\text { (post } \\
\text { add-on) }\end{array}$ \\
\hline 1 & $62 /$ male & adenocarcinoma & L858R & erlotinib (2nd-line)/PR & 251 days & pemetrexed/PR & $>176$ days \\
\hline 2 & 73/male & adenocarcinoma & L858R & erlotinib (2nd-line)/PR & 245 days & pemetrexed/PD & 56 days \\
\hline 3 & $65 /$ male & adenocarcinoma & unknown & erlotinib (5th-line)/SD & 784 days & pemetrexed/SD & $>105$ days \\
\hline Present case & $35 /$ male & adenocarcinoma & wild-type & gefitinib (1st-line)/PR & 223 days & gemcitabine + vinorelbine/PR & 141 days \\
\hline
\end{tabular}

EGFR-TKI = Epidermal growth factor receptor-tyrosine kinase inhibitor; $\mathrm{PD}=$ progressive disease; $\mathrm{PR}=$ partial response; $\mathrm{SD}=$ stable disease. 


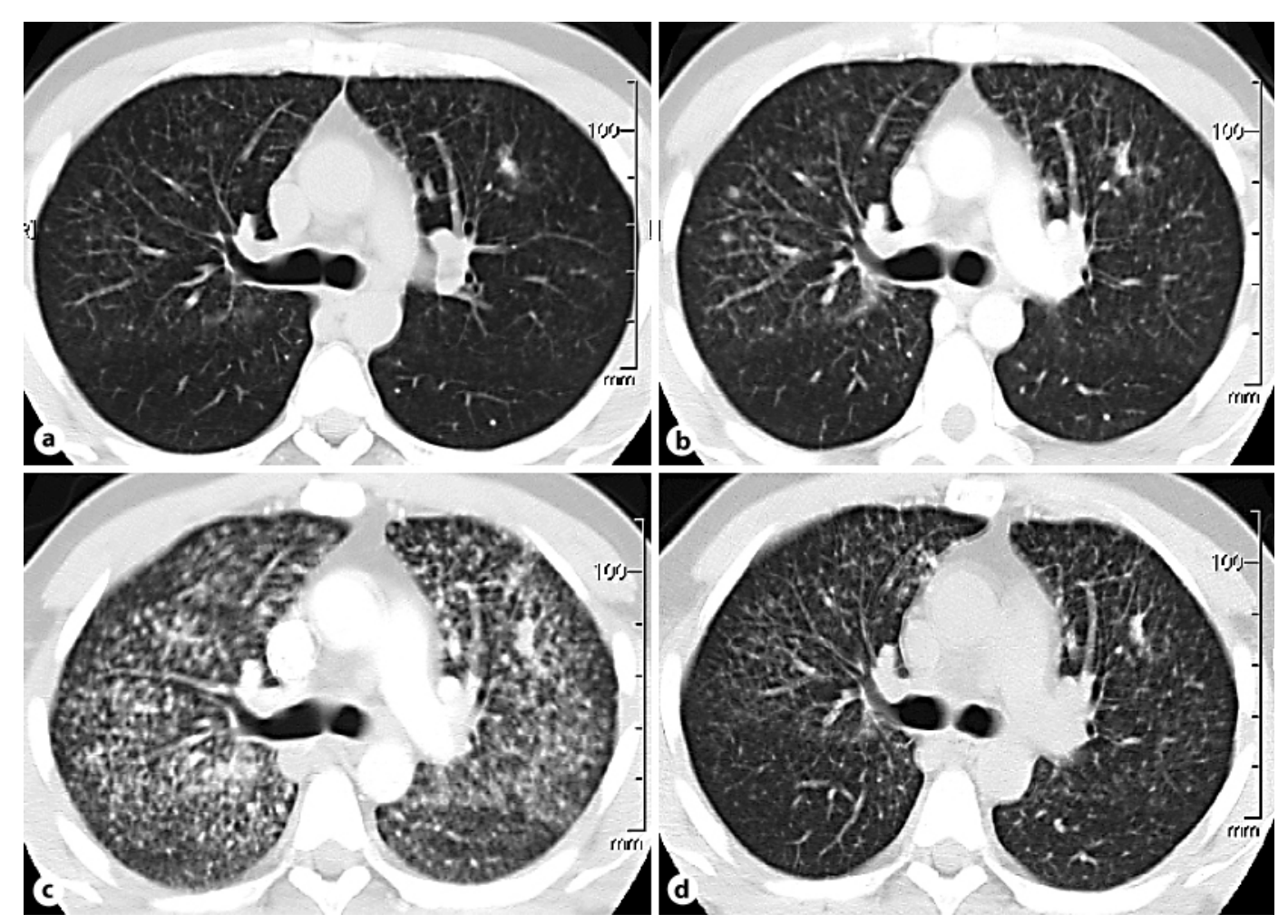

Fig. 1. CT of the chest at 2 months before PD (a), PD (b), 3 weeks after stopping gefitinib (c), and at 10 days after restarting gefitinib (d). 


\section{References}

1 Mok TS, Wu YL, Thongprasert S, Yang CH, Chu DT, Saijo N, et al: Gefitinib or carboplatin-paclitaxel in pulmonary adenocarcinoma. N Engl J Med 2009;361:947-957.

2 Riely GJ, Kris MG, Zhao B, Akhurst T, Minton DT, Moore E, et al: Prospective assessment of discontinuation and reinitiation of erlotinib or gefitinib in patients with acquired resistance to erlotinib or gefitinib followed by the addition of everolimus. Clin Cancer Res 2007;13:5150-5155.

3 Van Oosterom AT, Dumez H, Desai J, Stroobants S, Van Den Abbeele AD, Clement P, et al: Combination signal transduction inhibition: a phase I/II trial of the oral mTOR-inhibitor everolimus (E, RAD001) and imatinib mesylate (IM) in patients (pts) with gastrointestinal stromal tumor (GIST) refractory to IM. ASCO Meeting Abstracts 2004;22:3002.

-4 Giaccone G, Herbst RS, Manegold C, Scagliotti G, Rosell R, Miller V, et al: Gefitinib in combination with gemcitabine and cisplatin in advanced non-small-cell lung cancer: a phase III trial - INTACT 1. J Clin Oncol 2004;22:777-784.

-5 Herbst RS, Giaccone G, Schiller JH, Natale RB, Miller V, Manegold C, et al: Gefitinib in combination with paclitaxel and carboplatin in advanced non-small-cell lung cancer: a phase III trial-INTACT 2. J Clin Oncol 2004;22:785-794.

-6 Herbst RS, Prager D, Hermann R, Fehrenbacher L, Johnson BE, Sandler A, et al: TRIBUTE: a phase III trial of erlotinib hydrochloride (OSI-774) combined with carboplatin and paclitaxel chemotherapy in advanced nonsmall-cell lung cancer. J Clin Oncol 2005;23:5892-5899.

7 Gatzemeier U, Pluzanska A, Szczesna A, Kaukel E, Roubec J, De Rosa F, et al: Phase III study of erlotinib in combination with cisplatin and gemcitabine in advanced non-small-cell lung cancer: the Tarceva Lung Cancer Investigation Trial. J Clin Oncol 2007;25:1545-1552.

8 Yoshimura M, Nakamura S, Imamura F, Ueno K, Yamamoto S, Igarashi T, et al: Severe myelotoxicity in a combination of gefitinib and vinorelbine. Lung Cancer 2004;45:121-123.

-9 Pujol JL, Viens P, Rebattu P, Laurie SA, Feld R, Deneulin A, et al: Gefitinib (IRESSA) with vinorelbine or vinorelbine/cisplatin for chemotherapy-naive non-small cell lung cancer patients. J Thorac Oncol 2006;1:417424.

10 Davies AM, Ho C, Beckett L, Lau D, Scudder SA, Lara PN, et al: Intermittent erlotinib in combination with pemetrexed: phase I schedules designed to achieve pharmacodynamic separation. J Thorac Oncol 2009;4:862868.

11 Pao W, Chmielecki J: Rational, biologically based treatment of EGFR-mutant non-small-cell lung cancer. Nat Rev Cancer 2010;10:760-774. 